\title{
Smartphones jetzt noch smarter? - Möglichkeit des Einsatzes als „Dosiswarner“
}

\author{
Usability of Smartphones for Dose Alerts
}

Autoren

Institute
T. Kaireit ${ }^{1}$, G. Stamm ${ }^{1}$, C. Hoeschen ${ }^{2}$, F. K. Wacker ${ }^{1}$

Institut für Diagnostische und Interventionelle Radiologie, Medizinische Hochschule, Hannove

${ }^{2}$ Abt. Medizinische Strahlenphysik und Diagnostik, Helmholtz-Zentrum Neuherberg

\section{Key words}

- radiation safety

- technical aspects

- radiation

- technology assessment

eingereicht 31.7 .2012

akzeptiert $\quad 5.1 .2013$

Bibliografie

Dol http://dx.doi.org/

10.1055/s-0032-1330705

Online-Publikation: 2013

Fortschr Röntgenstr 2013; 185:

558-562 @ Georg Thieme

Verlag KG Stuttgart · New York . ISSN 1438-9029

\section{Korrespondenzadresse}

Dr. Georg Stamm

Institut für Diagnostische und

Interventionelle Radiologie,

Medizinische Hochschule

Carl-Neuberg-Str. 1

30625 Hannover

Germany

stamm.georg@mh-hannover.de Tel.: 05 11/532-2690

Fax: 05 11/532-38 88

\section{Zusammenfassung \\ $\nabla$}

Ziel: Die Möglichkeit, mit den in den Kameras von Smartphones verwendeten (CMOS-)Chips neben sichtbarem Licht auch andere elektromagnetische Strahlung zu registrieren, führte zu der Entwicklung von Apps zur Messung ionisierender Strahlung. Ziel der vorliegenden Studie war es, die Genauigkeit dieser Messmethode exemplarisch zu überprüfen und mögliche Anwendungsbereiche zu identifizieren.

Material und Methoden: Es wurden 2 Apps auf 2 Hardwareplattformen im Vergleich zu einem geeichten Ionisationsmessgerät und einem elektronischen Personendosimeter getestet. Zur Erstellung der Kalibrierungskurve dienten Dosisraten zwischen $12700 \mu \mathrm{Sv} / \mathrm{h}$ und 5,7 $\mu \mathrm{Sv} / \mathrm{h}$. Der Einsatz der Apps für die Messung der Streustrahlung eines C-Bogens erfolgte an einem Alderson-Rando-Phantom.

Ergebnisse: Während eine App sich als unbrauchbar erwies, ergaben sich bei der anderen folgende Messwerte: Die seitlich am Phantom gemessene Streustrahlung lag mit $117 \mu \mathrm{Sv} / \mathrm{h}$ (2 m Abstand) bis 5910 $\mu \mathrm{Sv} / \mathrm{h}(0,3 \mathrm{~m}$ Abstand) jeweils ca. um den Faktor 1,4 unterhalb der mit der Ionisationsmesskammer ermittelten Werte. Auf Höhe der Schilddrüse eines Untersuchers wurden $4200-4400 \mu \mathrm{Sv} / \mathrm{h}$ gemessen. Bei geringem Abstand zum Phantom zeigte sich eine starke Winkelabhängigkeit der Messergebnisse. In $0,3 \mathrm{~m}$ Abstand war bei einem Abkippen des Smartphones um $45^{\circ}$ nach unten eine Abnahme von $3000 \mu \mathrm{Sv} / \mathrm{h}$ auf $972 \mu \mathrm{Sv} / \mathrm{h}$, nach oben eine $\mathrm{Zu}-$ nahme auf $5000 \mu \mathrm{Sv} / \mathrm{h}$ zu verzeichnen. Im Abstand von $1 \mathrm{~m}$ war dieser Effekt deutlich schwächer.

Schlussfolgerung: Es konnte gezeigt werden, dass ein Nachweis ionisierender Strahlung mit Smartphone-Kameras prinzipiell möglich ist. Trotz überraschend guter Messergebnisse ist die Genauigkeit aufgrund starker Winkelabhängigkeit für die Personendosimetrie nicht ausreichend. Eine qualitative Aussage im Sinne eines Dosiswarners ist jedoch möglich.

\section{Abstract \\ v}

Purpose: Smartphone apps for measuring ionizing radiation use the capability of (CMOS) camera chips to detect not only perceivable light but also electromagnetic wave radiation. The present study evaluates the accuracy of hardware and software and defines possible applications for the detection of X-ray radiation fields.

Materials and Methods: 2 apps and 2 different devices were tested in comparison with a calibrated ionization chamber and a personal electronic dosimeter. A calibration curve was determined for dose rates between $12700 \mu \mathrm{Sv} / \mathrm{h}$ and $5.7 \mu \mathrm{Sv} / \mathrm{h}$ generated by a C-arm system.

Results: The measured scattered radiation produced by an Alderson-Rando phantom ranged from $117 \mu \mathrm{Sv} / \mathrm{h}$ (at a distance of $2 \mathrm{~m}$ ) to 5910 $\mu \mathrm{Sv} / \mathrm{h}$ (at a distance of $0.3 \mathrm{~m}$ ) and was 1.4 times less than the values of the ionization chamber. The exposure rate for the operator's thyroid was within $4200-4400 \mu \mathrm{Sv} / \mathrm{h}$. We found a strong dependence of the measurements on the angulation of the Smartphone, especially for short distances from the phantom (at a distance of $0.3 \mathrm{~m}, \mathrm{a} 45^{\circ}$ rotation downwards in a vertical direction caused a decrease from $3000 \mu \mathrm{Sv} / \mathrm{h}$ to $972 \mu \mathrm{Sv} / \mathrm{h}$, while an upwards rotation resulted in an increase to 5000 $\mu \mathrm{Sv} / \mathrm{h})$. For a distance of $1 \mathrm{~m}$, this effect was remarkably smaller.

Conclusion: Smartphones can be used to detect ionizing radiation but showed limited accuracy and are heavily dependent on the angulation of the device. Qualitative measurements and utilization for dose alerts are possible. 


\section{Einleitung \\ $\nabla$}

Die in der Röntgenverordnung vorgeschriebene amtliche Personendosimetrie hat derzeit einen nicht zu vernachlässigenden Anteil an den für Strahlenschutz aufgewendeten Mitteln (ca. $60 €$ pro überwachter Person und Jahr [1]). In Zeiten wachsenden Kostendrucks im Gesundheitssystem ist die Suche nach gut verfügbaren und kostengünstigen Alternativen daher sinnvoll.

Die Einführung von App-Stores für Smartphones (und TabletComputer) eröffnet die Möglichkeit, diese Geräte durch kleine Zusatzprogramme in ihrem Funktionsumfang zu erweitern. Das beständig wachsende Angebot maßgeschneiderter Apps sowie die mit der steigenden Verbreitung der Geräte fallenden Anschaffungspreise führen, bislang vor allem im privaten Umfeld, zu einer wachsenden Beliebtheit von Smartphones und TabletComputer. Diese Entwicklung macht auch vor medizinischen Fragestellungen nicht halt, zunehmend werden für eine Vielzahl von medizinischen Themen sogenannte „Medical Apps“ entwickelt [2-6]. Die Einsatzmöglichkeiten reichen von der Erfassung von Vitalparametern (z.B. Blutdruck und Körpertemperatur) über die Darstellung radiologischen Bildmaterials bis hin zur Visite mit dem Tablet-Computer. Bedingung für den professionellen Einsatz einer jeden Medical App, insbesondere wenn sie zur Diagnosefindung und therapeutischen Entscheidungen beiträgt, ist die Zulassung nach dem Medizinproduktegesetz (MPG) und die Erlangung einer CE-Kennzeichnung [7-11]. Ohne ein entsprechendes Zulassungsverfahren besteht die Gefahr haftungsrechtlicher Konsequenzen für Hersteller und Anwender [12].

Derzeit entwickeln verschiedene Programmierer Apps zur Messung ionisierender Strahlung ohne externe Hardware [13, 14].

Als Sensor wird der in der Kamera verbaute Complementary-Metal-Oxide-Semiconductor(CMOS)-Chip genutzt. Dieser kostengünstige Chiptyp wird in den Kameras fast jedes elektronischen Geräts verwendet, in Kombination mit Szintillatoren auch als Detektor in der Radiologie. CMOS-Photodioden lassen sich nicht nur durch sichtbares Licht, sondern auch durch andere elektromagnetische Wellenstrahlung anregen.

Ziel dieser Studie ist es, die erzielbare Messgenauigkeit dieses Funktionsprinzips gegenüber einem geeichten Ionisationskammer-Messgerät sowie die Nachweisbarkeit von Röntgenstreustrahlung in einem möglichst praxisnahen Szenario zu evaluieren. Zu diesem Zweck wurden 2 Apps auf 2 unterschiedlichen Plattformen getestet und auf Grundlage der Messergebnisse mögliche Anwendungsbereiche im Vergleich zu den etablierten elektronischen Dosimetern identifiziert [15].

\section{Material und Methoden}

$\nabla$

Für die Versuche wurden ein Smartphone (HUAWEI IDEOS X3 mit Android 2.3 Betriebssystem) mit der App „Radioactivity Counter“ sowie ein Apple iPad 2 in Verbindung mit der App „WikiSensor" ausgewählt.

Voraussetzung für die Detektion von ionisierender Strahlung ist die Abschirmung des Kamerasensors gegenüber sichtbarem Licht bei gleichzeitig möglichst geringer Abschwächung der hochenergetischen Strahlung durch das verwendete Abschirmmaterial (in unseren Versuchen ein geschwärzter Röntgenfilm). Das Aufleuchten einzelner Pixel des CMOS-Chips wird von der Programmroutine registriert und mittels einer Kalibrierungskurve in eine Dosisleistung umgerechnet. Für die App „Radioactivity Counter“ wurden bereits vom Programmierer für verschiedene
Smartphone-Modelle Kalibrierungskurven mit einem ${ }^{60} \mathrm{Co}-\mathrm{Strah}-$ ler erstellt. Im Hinblick auf die Messung von Röntgenstreustrahlung am Alderson-Rando-Phantom in einem OP-Szenario wurde mit einem C-Bogen (Exposkop CB 7-D, Ziehm Imaging, Nürnberg, Deutschland) bei einer Röhrenspannung von $70 \mathrm{kV}$ eine Kalibrierung durchgeführt ( $\bullet$ Tab.1), diese mit der originären Kalibrierungskurve verglichen und anschließend in den weiteren Versuchen genutzt. Das Smartphone und die Messkammer eines Ionisationsmessgerätes (TOL/F, Berthold, Bad Wildbad, Deutschland) wurden nacheinander auf einem Bucky-Tisch liegend in den direkten geschwächten Strahlengang des C-Bogens eingebracht und die vom Smartphone über 3 min gemittelten Ereignisse/min bei kontinuierlicher Röntgenstrahlung sowie die Dosisleistung mittels des TOL/F gemessen. Zur Abschwächung der Strahlung wurden eine $17 \mathrm{~cm}$ dicke Plexiglasschicht und bis $\mathrm{zu}$ vier $1 \mathrm{~cm}$ starke Aluminiumplatten genutzt. Für Messungen mit noch geringerer Dosisleistung wurde zusätzlich eine $2 \mathrm{~mm}$ Kupferplatte verwendet. Diese 6 Messungen wurden jeweils dreimal wiederholt, die Daten anschließend mittels Regression hinsichtlich ihrer Plausibilität überprüft und mit der bestehenden Kalibrierungskurve verglichen.

Nach Eingabe der errechneten Kalibrierungskurve in „Radioactivity Counter" wurde bei $70 \mathrm{kV}$ Röhrenspannung die Streustrahlung am Alderson-Rando-Phantom gemessen ( $\bullet$ Abb. 1). Dieses wurde auf Höhe der Darmbeinschaufel (Schicht 30 des Phantoms) mittig im senkrechten Zentralstrahl auf dem Tisch liegend positioniert. Als Messpunkte wurden seitlich am Phantom in Höhe Schicht 30 auf Mitte der dorsoventralen Achse Abstände von 0,3-2m zwischen Mittelpunkt der Transversalachse und

Tab. 1 Kalibrierung des Herstellers von Radioactivity Counter für ${ }^{60} \mathrm{Co}$ Strahlung [14] und die Mittelwerte der Kalibriermessungen bei 70 kV Röntgenstrahlung.

\begin{tabular}{|crlr|}
\hline Kalibrierung ${ }^{60} \mathrm{Co}$ & & Kalibrierung $70 \mathrm{kV}$ & \\
\hline Dosisleistung $(\mu \mathrm{Sv} / \mathrm{h})$ & $\mathrm{CPM}$ & Dosisleistung $(\mu \mathrm{Sv} / \mathrm{h})$ & \multicolumn{1}{c|}{$\mathrm{CPM}$} \\
\hline 50000 & 204133 & 50000 & 1233651 \\
\hline 1000 & 4118 & 10000 & 258436 \\
\hline 100 & 418 & 1000 & 27615 \\
\hline & & 100 & 2951 \\
\hline
\end{tabular}

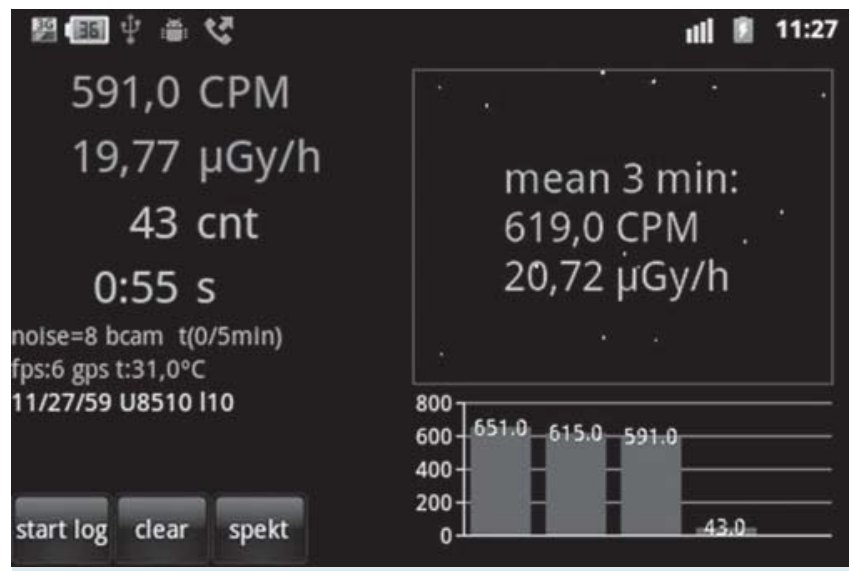

Abb. 1 Screenshot der Anzeige des Radioactivity Counters. Im Rechteck rechts oben werden neben dem Mittelwert über 3 je 1-minütige Messungen - auch die mit dem CMOS-Chip registrierten Einzelereignisse - als weiße Pixel angezeigt. 
Kamera/Messkammer (Strecke s in $\bullet$ Abb.2) ausgewählt. Das Smartphone wurde so positioniert, dass eine gedachte Linie zwischen dem Mittelpunkt der Linse und dem Referenzpunkt des Phantoms senkrecht auf Längs- und Querachse des verbauten CMOS-Chips steht ( $\bullet$ Abb. 2).

Zusätzlich wurde die Streustrahlung auf Höhe der Schilddrüse eines Untersuchers, d.h. in einem Abstand von ca. $50 \mathrm{~cm}$ unter einem Winkel von $45^{\circ}$ ausgehend von der Körpermitte (transversale Achse) des Phantoms, gemessen. Nacheinander wurden Smartphone („RadioactivityCounter“), iPad („WikiSensor“), TOL/ F sowie ein Personendosimeter (EPD Mk 2, Thermo Fischer Scientific, Erlangen, Deutschland) mittels eines Stativs an den Messpunkten positioniert und unter Durchleuchtung die Streustrahlung gemessen. Über einen Zeitraum von $3 \mathrm{~min} /$ Messung wurde die gemessene Dosisleistung von den Apps gemittelt. Zur Bestimmung der Winkelabhängigkeit der Messergebnisse des AndroidSmartphones wurde seitlich am Phantom in Höhe Schicht 30 auf Mitte der dorsoventralen Achse im Abstand 0,3 und $1 \mathrm{~m}$ die Messung wiederholt und dabei das Mobiltelefon um $45^{\circ}$ nach links/ rechts gedreht, respektive um $45^{\circ}$ nach oben/unten gekippt am Stativ befestigt.

Die App „WikiSensor“ (iPad) sieht keine eigene Möglichkeit zur Kalibrierung vor. Die beschriebenen Versuche zur Messung der Streustrahlung am Phantom wurden bei unrealistischen Messergebnissen vorzeitig abgebrochen und es wurden keine weiteren Versuche mit dem iPad durchgeführt.

\section{Ergebnisse}

In insgesamt 3 Versuchsreihen zur Erstellung der Kalibrierungskurve wurden mit dem Ionisationskammer-Messgerät Dosisraten zwischen $12700 \mu \mathrm{Sv} / \mathrm{h}(17 \mathrm{~cm}$ Plexiglas) und 5,7 $\mathrm{SSv} / \mathrm{h}$ (17 cm Plexiglas, $4 \mathrm{~cm} \mathrm{Al}, 2 \mathrm{~mm} \mathrm{Cu}$ ) gemessen. Das erhaltene Datenmaterial zeigt einen exponentiellen Zusammenhang zwischen den gemessenen Ereignissen/min (CPM) und der Dosisleistung ( $\mathrm{DL}=33,897 \times$ Ereignisse $^{0,971}$ ) in einem Messbereich zwischen $69 \mu \mathrm{Sv} / \mathrm{h}$ und $12700 \mu \mathrm{Sv} / \mathrm{h}$ ( Abb.3) bei einer Messunsicherheit von ca. $9 \%$ Die bei dem Smartphone stark schwankenden Messwerte unterhalb von $50 \mu \mathrm{Sv} / \mathrm{h}$ wurden für die weitere Betrachtung nicht berücksichtigt (hierzu hätten die Messzeiten auf unrealistische hohe Werte gesetzt werden müssen).

Bei Bestimmung der seitlich austretenden Streustrahlung am Alderson-Rando-Phantom wurden mit dem TOL/F Dosisleistungen zwischen $117 \mu \mathrm{Sv} / \mathrm{h}$ (2 m Abstand) und $5910 \mu \mathrm{Sv} / \mathrm{h}(0,3 \mathrm{~m}$ Abstand) gemessen. Die Abstandsabhängigkeit der Messwerte folgt in etwa dem Abstandsquadratgesetz. Die mit „Radioactivity Counter“ ermittelten Dosisleistungen lagen jeweils ca. um den Faktor 1,4 abweichend unter den mit dem TOL/F gemessenen Werten ( $\bullet$ Abb.4).

Auf Höhe der Schilddrüse eines Untersuchers wurden 4200 $4400 \mu \mathrm{Sv} / \mathrm{h}$ (TOL/F $4400 \mu \mathrm{Sv} / \mathrm{h}$; , Radioactivity Counter“ $4400 \mu \mathrm{Sv} /$ h; EPD Mk2 $4200 \mu \mathrm{Sv} / \mathrm{h}$ ) gemessen.

Gerade bei geringem Abstand zum Phantom zeigte sich eine starke Winkelabhängigkeit der Messergebnisse des Smartphones: Im Abstand von 0,3 m führte ein Abkippen des Smartphones um $45^{\circ}$ nach unten zu einer Abnahme der gemessenen Dosisleistung von $3000 \mu \mathrm{Sv} / \mathrm{h}$ auf $972 \mu \mathrm{Sv} / \mathrm{h}$, wohingegen ein Abkippen um $45^{\circ}$ nach oben zu einer Zunahme auf $5000 \mu \mathrm{Sv} / \mathrm{h}$ führte ( $\bullet$ Abb.5). Im Abstand von $1 \mathrm{~m}$ verringerte sich sowohl bei Abkippen als auch bei Drehungen stets die ermittelte Dosisleistung ( $\bullet$ Abb.5).

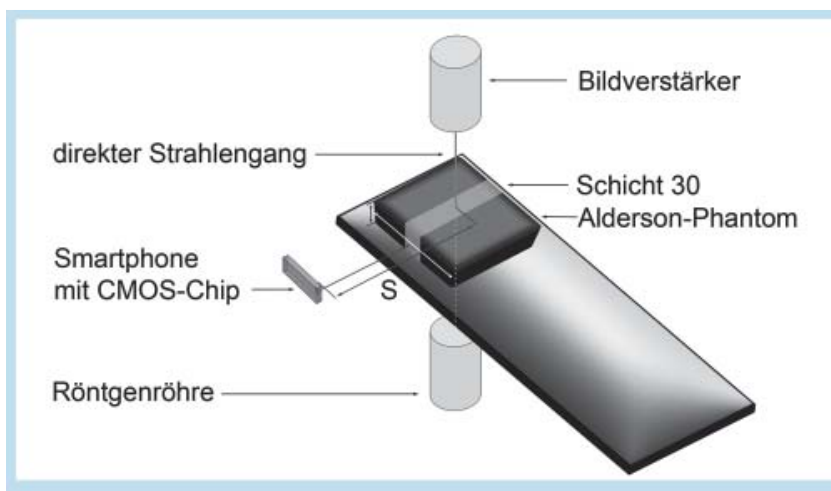

Abb. 2 Schematischer Versuchsaufbau für die Messung der Streustrahlung mit dem Smartphone. Bei der Kalibriermessung wurde das AldersonRando-Phantom durch Plexiglasplatten ersetzt, Smartphone bzw. Ionisationskammer befanden sich im direkten geschwächten Strahlengang.

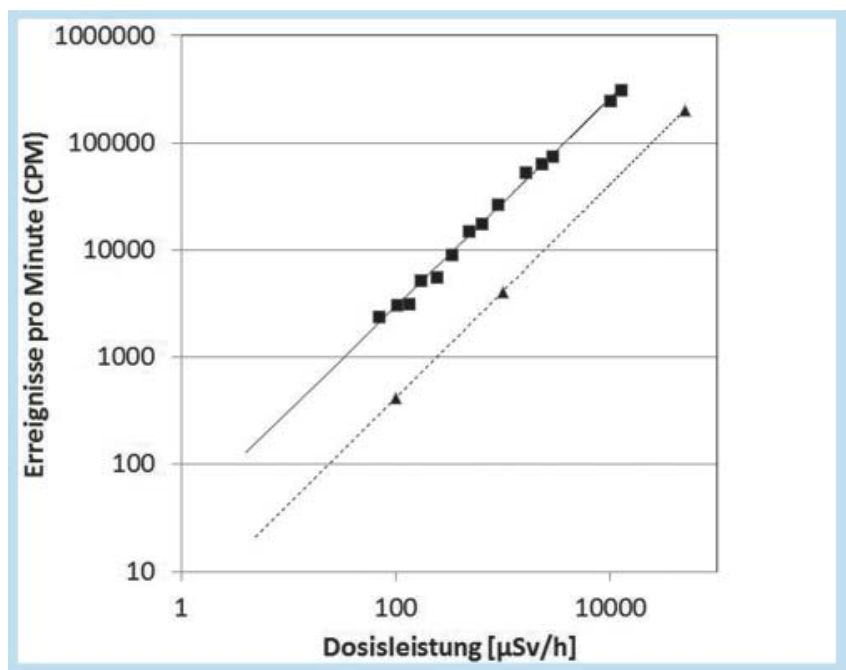

Abb. 3 Kalibriermessung des Smartphones bei 70 kV Röntgenstrahlung (durchgezogene Linie/ $\mathbf{\square}$ ). Die Abweichung zur Kalibrierkurve aus dem ${ }^{60} \mathrm{Co}$-Messungen des Herstellers (gestrichelte Linie/ $\mathbf{\Lambda}$ ) ist auf die unterschiedliche Strahlenqualität zurückzuführen.

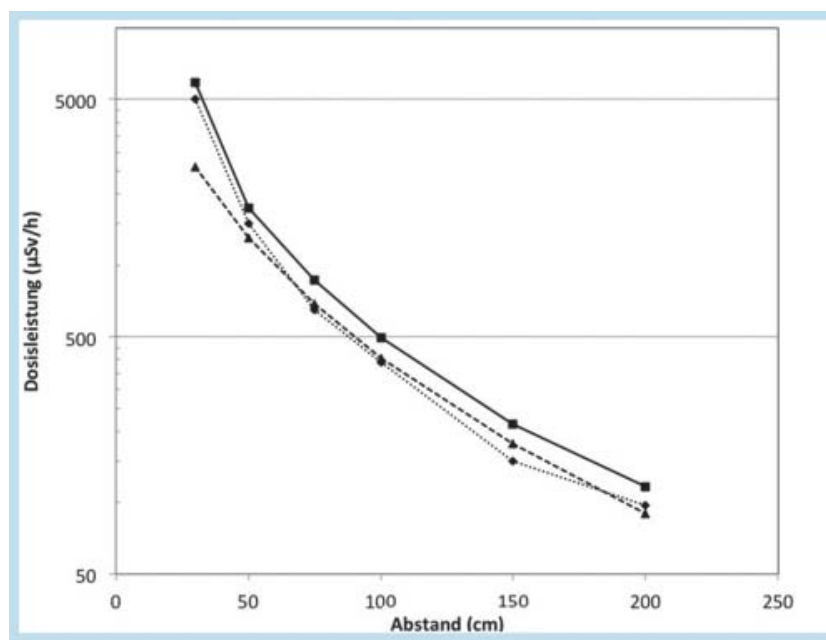

Abb. 4 Mittelwerte registrierter Streustrahlung am Alderson-RandoPhantom in verschiedenen Abständen $(30,50,75,100,150,200 \mathrm{~cm})$. TOL/F (durchgezogene Linie/ $\mathbf{\varpi})$, Smartphone (gestrichelte Linie/ $\bullet$ ) und EPD Mk2 (gepunktete Linie/ $\mathbf{\Delta}$ ). 


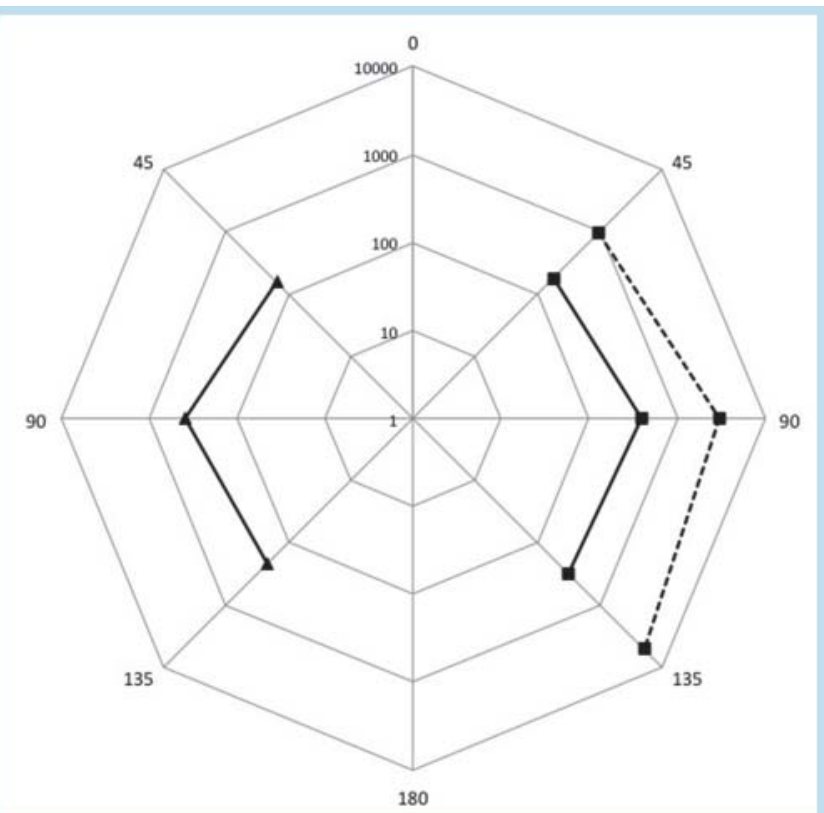

Abb.5 Winkelabhängigkeit der Messung der Streustrahlung mit dem Smartphone. Dabei entspricht ein Winkel von $90^{\circ}$ einem senkrechten Auftreffen der Strahlung auf die Kameralinse. Drehung um jeweils $45^{\circ}$ nach li. $\left(135^{\circ}\right)$ und re. $\left(45^{\circ}\right)$ in $1 \mathrm{~m}$ Abstand (durchgezogene Linie/^); Kippung um jeweils $45^{\circ}$ nach oben und unten in $1 \mathrm{~m}$ Abstand (durchgezogene Linie/ $\mathbf{\square}$ ) und in $30 \mathrm{~cm}$ Abstand (gestrichelte Linie/ $\mathbf{\square}$ ).

Die Messwerte der App „WikiSensor“ stellten sich schon während der Durchführung des Versuchs als inadäquat heraus: Bei einem Abstand von $50 \mathrm{~cm}$ vom Streukörper wurden 4483 Ereignisse/min bzw. laut App 58,3 $\mu \mathrm{Sv} / \mathrm{h}$ (TOL/F: $1750 \mu \mathrm{Sv} / \mathrm{h}$ ), in $75 \mathrm{~cm}$ Abstand 2532 Ereignisse/min bzw. 55,3 $\mu \mathrm{Sv} / \mathrm{h}$ (TOL/F: $865 \mu \mathrm{Sv} / \mathrm{h}$ ) gemessen. Daraufhin wurde die Messreihe abgebrochen.

\section{Diskussion}

$\nabla$

Die vorliegende Studie zeigt die prinzipielle Möglichkeit des Nachweises ionisierender Strahlung mittels CMOS-basierten Handy-Kameras, es müssen jedoch zahlreiche Fallstricke beachtet werden.

\section{Software}

Voraussetzung für eine quantitative Messung ionisierender Strahlung ist die Kalibrierung des jeweiligen Geräts. Die Messungen mit der nicht kalibrierbaren App „WikiSensor“ für das iPad 2 lieferten deshalb auch keine adäquaten Werte.

Ist die Kalibrierung möglich, muss diese für jedes Gerät, mindestens aber jede Gerätegeneration/jeden CMOS-Chiptyp und Version der Firmware/des Betriebssystems erfolgen, um konstruktive und bauteilspezifische Einflussgrößen zu korrigieren.

\section{Hardware}

Ist die softwareseitige Möglichkeit zur Kalibrierung gegeben („RadioactivityCounter“), kann die Dosisleistung gerichteter ionisierender Strahlung, die senkrecht auf den Kamera-Sensor trifft, in einem Bereich von ca. $100-3000 \mu \mathrm{Sv} / \mathrm{h}$ qualitativ gut beurteilt werden. Trifft Streustrahlung unter einem anderen Winkel auf den CMOS-Chip, ist eine zuverlässige Bestimmung nicht mehr möglich. Eine Erklärung bietet die Konstruktion der Kamera: Der Kamerasensor eines Smartphones misst ca. 9-10 $\mathrm{mm}^{2}$, die einzelnen Pixel weisen eine prismatische Struktur auf. Unterschiedliche Einstrahlwinkel hochenergetischer Strahlung können so entweder zu einer Anregung mehrerer Pixel, d. h. zu falsch hohen oder bei asymmetrischem Aufbau des Gehäuses unterschiedlich geschwächt und damit zu falsch niedrigen Messergebnissen führen. Eine weitere Einflussgröße ist die ungleichmäßige abstandsabhängige räumliche Verteilung der Streustrahlung. Bei der Untersuchung der Winkelabhängigkeit fanden wir im Vergleich zur senkrecht auftreffenden Streustrahlung sowohl eine Über- als auch eine Unterschätzung. Damit ist eine zuverlässige Messung der Streustrahlung bei nicht ebener Ausbreitung des Strahlenfelds und nicht senkrechtem Auftreffen auf die Linse grundsätzlich nicht möglich.

Ein weiterer Beleg hierfür ist die unterschiedliche Abweichung zwischen den Messwerten des ToL/F und des Smartphones bei den verschiedenen Expositionsbedingungen (seitlich Faktor 1,4 und auf Höhe der Schilddrüse Faktor 1) trotz der zuvor durchgeführten Kalibrierung. Neben dem Einfluss der Winkelabhängigkeit ist dies aus unserer Sicht auf die unterschiedlichen Strahlenqualitäten bei Messung und Kalibrierung und das sehr unterschiedliche aktive Detektorvolumen (Ionisationskammer versus HalbleiterChip) zurückzuführen.

\section{Firmware}

Die Firmware der Handykamera, insbesondere die Algorithmen zur Rauschunterdrückung, beeinflusst das Messergebnis maßgeblich. Mittels einer statistischen Analyse der Signale könnte die Genauigkeit erhöht und die Winkelabhängigkeit u. U. minimiert werden.

\section{CMOS-basierte externe Sensoren}

Eine Möglichkeit, die konstruktiv bedingten Messungenauigkeiten zu umgehen, bietet die Entwicklung von externen Sensoren. Da das Smartphone in diesem Fall nur noch zur Darstellung, Auswertung und Protokollierung der extern ermittelten Daten genutzt wird, werden Einflussgrößen wie die Bauform des Gehäuses und die Bildverarbeitung durch Firmware/Betriebssystem eliminiert. Die Erstellung einer Kalibrierungskurve müsste nur einmalig erfolgen, könnte direkt im Sensor hinterlegt und damit der Kalibrieraufwand deutlich reduziert werden. Die vorliegende Studie zeigt, dass dazu auch kostengünstige CMOS-Chips verwendet werden können. Zwingend erforderlich ist die Auswahl einer geeigneten Schnittstelle (Bluetooth, WLAN, Mikrofoneingang, USB) zur Kopplung von externer Hardware und App. Dies setzt bei einigen Modellen die Teilnahme an Entwicklerprogrammen und die (zum Teil teure) Lizenzierung der Schnittstelle voraus [17].

Mit externen Sensoren wäre sogar eine gleichzeitige Erfassung und Anzeige der Strahlenexposition an unterschiedlichen Messpunkten z. B. Schilddrüse und Oberkörper oder aber die Echtzeitüberwachung mehrerer Personen im Kontrollbereich möglich. Obwohl Smartphones unter „Idealbedingungen“ über die internen Kamerasensoren eine vorhandene ionisierende Strahlung überraschend genau nachweisen können, erachten wir aufgrund der in der Arbeit aufgezeigten Limitationen wie Winkelabhängigkeit und lange Messzeiten ( 3 min und mehr bei niedrigen Dosisleistungen) lediglich einen Einsatz als „Dosis-,, oder „Strahlungswarner“ als sinnvoll - nicht aber als Personendosimeter. Selbst für die schon länger etablierten elektronischen Dosimeter wie das verwendete EPD Mk2 waren aufwendige Studien notwendig, 
um ihre Eignung für die Personendosimetrie in der Röntgendiagnostik abzusichern und eine Bauartzulassung für definierte Bedingungen zu erhalten [16].

Im klinischen Alltag werden Smartphones und Tablet-Computer als Plattform zur mobilen Datenabfrage, -eingabe und, insbesondere im Bereich der ambulanten Patientenversorgung, Akquisition von Messdaten eine wichtige Rolle spielen. Inwieweit durch zukünftige Entwicklungen von externen Sensoren eine gleichzeitige verlässliche Erfassung und Anzeige einer Strahlenexposition möglich wird, muss noch abgewartet werden.

\section{Abkürzungen \\ App Application (kleines Softwareprogramm) \\ CMOS Complementary Metal Oxide Semiconductor \\ CPM Counts per minute \\ USB Universal Serial Bus \\ WLAN Wireless Local Area Network}

\section{Literatur}

1 MPA Dortmund. Preisliste. http://www.mpanrw.eu/downloads/ informationsportal-der-personendosis-messstelle/ (abgerufen am 25.6.2012)

2 MIM Software Inc. Mobile MIM. http://itunes.apple.com/us/app/ mobile-mim/id281922769? mt=8 (abgerufen am 25.6.2012)

3 Aycan Digitalsysteme GmbH. aycan-mobile. www.aycan.de/produkte/ aycan-mobile.html (abgerufen am 25.6.2012)

4 Krüger-Brand $H$. Smartphones und Tablet-PCs im Gesundheitswesen: Strategien für mobile Anwendungen. Dtsch Arztebl 2011; 108: 45
5 Mißlbeck A. Der Doktor und das iPad: Neurologen online. http://www.aerztezeitung.de/praxis_wirtschaft/klinikmanagement/ article/679723/doktor-ipad-neurologen-online.html (abgerufen am 25.6.2012)

6 Pramann O, Gärtner A, Albrecht UV. Medical Apps: Mobile Helfer am Krankenbett. Dtsch Arztebl 2012; 109: 22 -23, A-1201

7 Bundesministerium für Justiz. Gesetz über Medizinprodukte. www.gesetze-im-internet.de/mpg/index.html (abgerufen am 25.6.2012)

8 Deutsches Institut für Medizinische Dokumentation und Information. www.dimdi.de/static/de/mpg/recht/index.htm (abgerufen am 25.6.2012)

9 Deutsch E, Spickhoff A. Medizinrecht. 6. Aufl, Rn. 1636, 16392008

10 Gärtner A. Mobilgeräte und Apps in der Medizin aus regulatorischer Sicht. www.e-health-com.eu/fileadmin/user_upload/dateien/ Downloads/Gaertner_Mobilgeraete_und_Apps_aus_ regulatorischer_Sicht.pdf (abgerufen am 25.6.2012)

11 Jefferson E. FDA clears first diagnostic radiology application for mobile devices, FDA NEWS RELEASE 2011. www.fda.gov/NewsEvents/ Newsroom/PressAnnouncements/ucm242295.htm (abgerufen am 25.6.2012)

12 Praman O, Graf K, Albrecht U-V. Tablet-PCs im Krankenhaus. Hygienische Aspekte beachten. Dtsch Arztebl 2012; 109: 14, A-7067

13 NORM INDUSTRIES. WikiSensor. http://wikisensor.com/ (abgerufen am 25.6.2012)

14 Klein R. Radioactivity Counter. München: http://www.hotray-info.de/ (abgerufen am 25.6.2012)

15 Ambrosi P, Borowski M, Iwatschenko M et al. Considerations concerning the use of counting active personal dosimeters in pulsed fields of ionising radiation. Radiation Protection Dosimetry 2010; 139 (4): 483 493

16 Borowski M, Poppe B, Looe HK et al. Sind elektronische Personendosimeter in klinischen Expositionssituationen grundsätzlich nicht einsetzbar? Fortschr Röntgenstr 2010; 182: $773-779$

17 Apple. MFi Program. https://developer.apple.com/programs/mfi/ (abgerufen am 25.6.2012) 